\title{
Exotic criticality in the dimerized spin-1 $X X Z$ chain with single-ion anisotropy
}

\author{
Satoshi Ejima ${ }^{*}$, Tomoki Yamaguchi ${ }^{2}$, Fabian H. L. Essler ${ }^{3}$, Florian Lange ${ }^{1}$, Yukinori Ohta ${ }^{2}$, \\ Holger Fehske ${ }^{1}$ \\ 1 Institute of Physics, University Greifswald, 17489 Greifswald, Germany \\ 2 Department of Physics, Chiba University, Chiba 263-8522, Japan \\ 3 The Rudolf Peierls Centre for Theoretical Physics, Oxford University, Oxford OX1 3NP, \\ UK \\ * ejima@physik.uni-greifswald.de
}

April 7, 2022

\begin{abstract}
We consider the dimerized spin-1 $X X Z$ chain with single-ion anisotropy $D$. In absence of an explicit dimerization there are three phases: a large- $D$, an antiferromagnetically ordered and a Haldane phase. This phase structure persists up to a critical dimerization, above which the Haldane phase disappears. We show that for weak dimerization the phases are separated by Gaussian and Ising quantum phase transitions. One of the Ising transitions terminates in a critical point in the universality class of the dilute Ising model. We comment on the relevance of our results to experiments on quasi-one-dimensional anisotropic spin-1 quantum magnets.
\end{abstract}

\section{Contents}

1 Introduction $\quad 2$

2 Ground-state phase diagram 3

3 Field theory approach

3.1 Renormalization group analysis 6

3.2 Quantum phase transitions 7

3.2.1 $\mathrm{D}-\mathrm{LD} \leftrightharpoons \mathrm{D}-\mathrm{AFM}$ phase transition line 7

3.2.2 D-H $\leftrightharpoons \mathrm{D}-\mathrm{AFM}$ phase transition line 7

3.2.3 D-H $\leftrightharpoons \mathrm{D}-\mathrm{LD}$ phase transition line 7

4 DMRG analysis

4.1 Phase transition lines 8

4.1.1 D-LD $\leftrightharpoons \mathrm{D}-\mathrm{AFM}$ and $\mathrm{D}-\mathrm{H} \leftrightharpoons \mathrm{D}$-AFM Ising phase transition lines 8

4.1.2 $\mathrm{D}-\mathrm{H} \leftrightharpoons \mathrm{D}-\mathrm{LD}$ phase transition line 9

4.2 Topological order parameters 10

5 Relevance to experiments 11 
6 Summary and Conclusions 12

A Low-energy projections of operators 13

A.1 Integrating out the bosonic degrees of freedom 13

A.2 Integrating out the fermionic degrees of freedom 14

B Ground-state phase diagram for strong dimerization 15

$\begin{array}{ll}\text { References } & 16\end{array}$

\section{Introduction}

It is well established that quantum effects in one-dimensional antiferromagnetic (AFM) spin systems lead to interesting physical phenomena. While a uniform Heisenberg chain is gapless for half-integer spins, an exotic ground state with a finite gap appears for integer spins 1 . For spins $S=1$, this Haldane phase can be understood in the framework of the Affleck-KennedyLieb-Tasaki model [2, 3], whose exact ground state can be constructed in terms of valence bonds, i.e., singlet pairs of $S=1 / 2$ spins. Meanwhile, the Haldane phase is recognized as a symmetry-protected topological (SPT) state [4,5] and attracts continued attention from both theoretical and experimental points of view. For instance, the Haldane gap was confirmed experimentally in a compound with $\mathrm{Ni}^{2+}$ ions $\mathrm{Ni}\left(\mathrm{C}_{2} \mathrm{H}_{8} \mathrm{~N}_{2}\right)_{2} \mathrm{NO}_{2}\left(\mathrm{ClO}_{4}\right)[6$, 7, in which a small value of the single-ion anisotropy $D$ was reported [8]. A minimal model for the description of such anisotropic spin-1 chains is

$$
\hat{H}_{X X Z, D}=J \sum_{j}\left(\hat{\boldsymbol{S}}_{j} \cdot \hat{\boldsymbol{S}}_{j+1}\right)_{\Delta}+D \sum_{j}\left(\hat{S}_{j}^{z}\right)^{2}
$$

where $\left(\hat{\boldsymbol{S}}_{j} \cdot \hat{\boldsymbol{S}}_{j+1}\right)_{\Delta}=\hat{S}_{j}^{x} \hat{S}_{j+1}^{x}+\hat{S}_{j}^{y} \hat{S}_{j+1}^{y}+\Delta \hat{S}_{j}^{z} \hat{S}_{j+1}^{z}$. Assuming a positive exchange parameter $J>0$ and $\Delta>0$, the ground-state phase diagram exhibits three gapped phases [9]. At the isotropic point $(D=0$ and $\Delta=1)$ the model is in a Haldane phase. A sufficiently strong single-ion anisotropy $D / J$ induces a Gaussian quantum phase transition (QPT) with central charge $c=1$ to a topologically trivial large- $D$ (LD) phase. On the other hand, increasing $\Delta$ for fixed $D=0$ from the isotropic point leads to a Ising QPT with $c=1 / 2$ to a long-range ordered AFM phase. At larger values of $\Delta$ and $D$ there is a first order transition between the LD and AFM phases.

A natural extension of the spin- $1 X X Z$ chain (1) is the introduction of an explicit bond alternation

$$
\hat{H}=\hat{H}_{X X Z, D}+J \sum_{j} \delta(-1)^{j}\left(\hat{\boldsymbol{S}}_{j} \cdot \hat{\boldsymbol{S}}_{j+1}\right)_{\Delta}
$$

Interestingly this model realizes dimerized versions of the same three phases as the one described by Eq. (1), namely, dimerized Haldane (D-H), AFM (D-AFM) and LD (D-LD) phases. The case $D=0$ has been studied previously 10,11 and it was found that the $\mathrm{D}-\mathrm{H}$ to D-LD transition is again of the Gaussian type, but the entire D-AFM-phase boundary, including 
the transition to the D-LD phase, belongs to the Ising universality class. A key question is how the criticality at the phase boundary changes, if both $D$ and $\delta$ are finite. Earlier studies of half-filled Hubbard-type models realizing SPT insulating and long-range ordered (chargedensity-wave) phases [12 14] indicated a transition line that is separated into continuous Ising and first-order QPTs. The meeting point of these lines belongs to the tricritical Ising universality class with $c=7 / 10$, which can be described by the second minimal model of conformal field theory [15, 16].

In this paper, we determine and analyze the ground-state phase diagram of the extended model (2) by means of field theory and matrix-product-state based density-matrix renormalization group (DMRG) 17, 18 techniques, focusing on the quantum criticality at the phase boundaries. By calculating the central charge $c$, we provide compelling evidence for the existence of a critical point in the tricritical Ising universality class. Field-theory predictions for the phases and the nature of the phase boundaries of the model (2) with both single-ion anisotropy $D$ and bond alternation $\delta$ are shown to be in excellent agreement with numerical simulations. Finally, we discuss the relevance of our results to experiments on dimerized spin-1 materials [19].

\section{Ground-state phase diagram}

Let us first describe the numerical method we have used to determine the phase boundaries of the model (2). By means of the infinite DMRG (iDMRG) 20] a characteristic correlation length $\xi_{\chi}$ can be calculated. While this $\xi_{\chi}$ is always finite for fixed bond dimension $\chi$, it strongly peaks at a critical point and therefore allows for an accurate determination of QPT points. This approach was already applied to half-filled Hubbard-type models [12 14$]$.

In order to identify the different continuous phase transitions occurring in the model (2), we calculate the corresponding central charges $c$ via the entanglement entropy. For a critical system with $L$ sites and periodic boundary conditions, the von Neumann entanglement entropy of a contiguous block of $\ell$ sites with the rest of the system is $S_{L}(\ell)=\frac{c}{3} \ln \left[\frac{L}{\pi} \sin \left(\frac{\pi \ell}{L}\right)\right]+s_{1}$, where $s_{1}$ is a non-universal constant 21]. An accurate determination of the central charge is possible by using the relation 13,22

$$
c(L) \equiv \frac{3\left[S_{L}(L / 2-2)-S_{L}(L / 2)\right]}{\ln \{\cos [\pi /(L / 2)]\}},
$$

where in view of the explicit dimerization the doubled unit cell has been taken into account.

Figure 1(a) shows the ground-state phase diagram of the model (2) for $\delta=0.1$. For weak dimerization, the D-H phase survives between the D-LD and D-AFM phases. In contrast to the model without dimerization, however, the transition between the D-LD and D-AFM phases is continuous below a critical end point $\left(\Delta_{\mathrm{c}}, D_{\mathrm{c}}\right) \simeq(3.90,3.64)$. Like the $\mathrm{D}-\mathrm{H} \leftrightharpoons \mathrm{D}$-AFM line, this part of the transition belongs to the Ising universality class with central charge $c=1 / 2$, except for the critical end point, which belongs to the universality class of the tricritical Ising model with $c=7 / 10$. A tricritical Ising point at which the transition becomes first order is not observed in the dimerized model without single-ion anisotropy, simply because in this case the transition between the D-LD and D-AFM phases is always continuous. At the phase boundaries involving the Haldane phase, the universality classes are the same as in the nondimerized model. Now the tricritical point, where the Haldane phase vanishes, is at $\left(\Delta_{\mathrm{tr}}\right.$, $\left.D_{\text {tr }}\right) \simeq(2.64,2.28)$. For $\delta \neq 0$, the central charge at this point is $c=1$. 

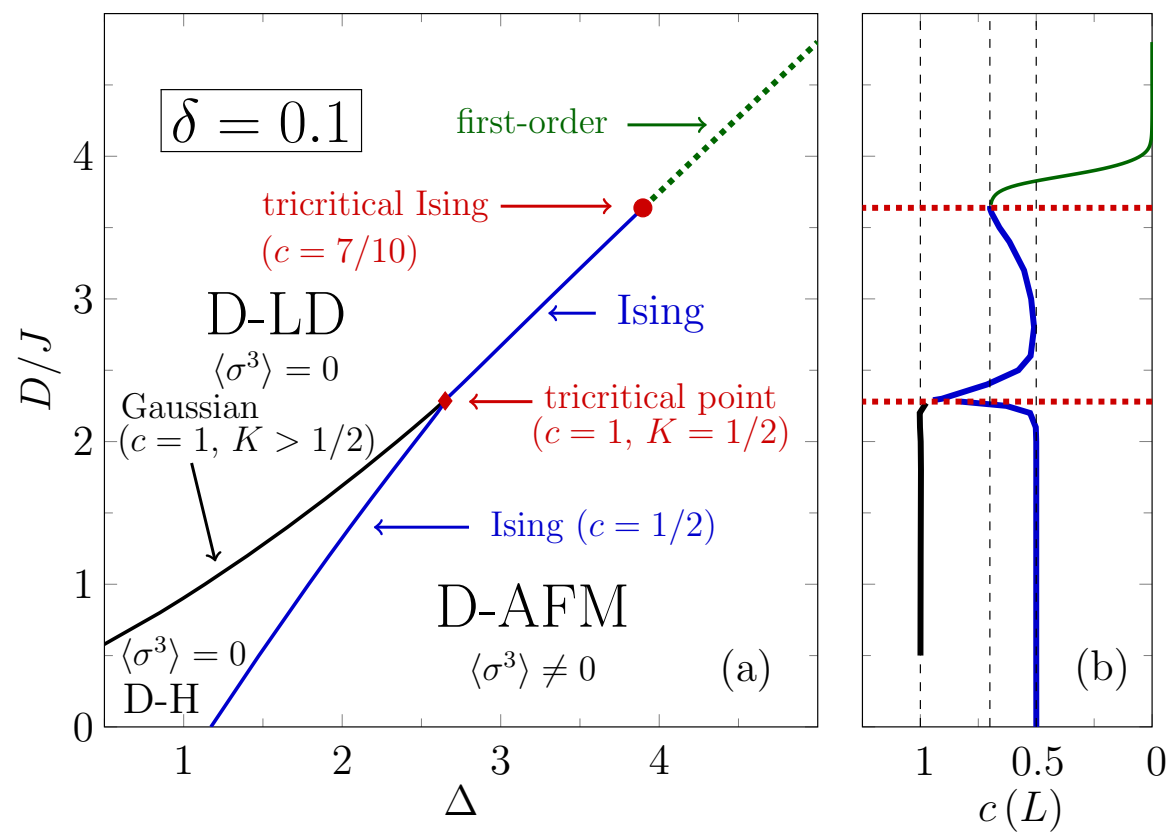

Figure 1: (a): Ground-state phase diagram of the model (2) for $\delta=0.1$. (b): Numerically obtained central charge $c(L)$ on various phase transition lines from Eq. (3) with $L=128$ and periodic boundary conditions.

In the following, combining field theory and DMRG, we discuss various QPTs, including the direct Ising transition from the D-LD to the D-AFM phase.

\section{$3 \quad$ Field theory approach}

In order to obtain a field-theory description of the model in the vicinity of the various phase transition lines we consider the Hamiltonian

$$
\hat{H}_{\mathrm{FT}}=\hat{H}-J \sum_{j}(1-\alpha)\left(\hat{\boldsymbol{S}}_{j} \cdot \hat{\boldsymbol{S}}_{j+1}\right)_{\Delta^{\prime}}^{2},
$$

which differs from Eq. (2) by an additional biquadratic exchange term. A field-theory description of the model (4) can be constructed in the vicinity of the Takhtajan-Babujian point [23 24] $\left(\alpha=0, \delta=0, D=0, \Delta=1\right.$ and $\left.\Delta^{\prime}=1\right)$ following Ref. [25]. This leads to a Hamiltonian density of the form

$$
\hat{\mathcal{H}}=\sum_{a=1}^{3} \frac{\mathrm{i} v_{a}}{2}\left[\hat{L}_{a} \partial_{x} \hat{L}_{a}-\hat{R}_{a} \partial_{x} \hat{R}_{a}\right]-\mathrm{i} m_{a} \hat{R}_{a} \hat{L}_{a}+\sum_{a=1}^{3} g_{a} \hat{J}^{a} \hat{J}^{a}+\lambda \hat{\sigma}^{1} \hat{\sigma}^{2} \hat{\sigma}^{3},
$$

where $\hat{L}_{a}$ and $\hat{R}_{a}$ are left and right moving Majorana fermions, $\hat{\sigma}^{a}$ are three Ising order parameter fields and

$$
\hat{J}^{a}=-(\mathrm{i} / 2) \epsilon^{a b c}\left[\hat{L}_{b} \hat{L}_{c}+\hat{R}_{b} \hat{R}_{c}\right]
$$

The parameter $\lambda$ in $\hat{\mathcal{H}}$ is proportional to the dimerization $\delta$ and by virtue of the $\mathrm{U}(1)$ symmetry of the microscopic Hamiltonian (4) we have $v_{1}=v_{2}, m_{1}=m_{2} \equiv m$, and $g_{1}=g_{2} \equiv g$. The 
masses $m$ and $m_{3}$ are functions of $D$ and $\alpha$. The functional form of this dependence is only known in the vicinity of the Takhtajan-Babujian point and in what follows we therefore take $m_{3}$ and $m$ as free parameters, which we adjust in order to recover the structure of the phase diagram obtained by DMRG. Our main working assumption is that the field theory (4) remains a good description of the low-energy degrees of freedom in the vicinity of the various phase transition lines in the microscopic model even far away in parameter space from the Takhtajan-Babujian point. We note that an alternative way of deriving a field theory proposed by Schulz [26] leads to equivalent results. A third approach would be to develop a field-theory description around the SU(3) symmetric point of the spin-1 chain [27 [30], but we do not pursue this here. The relation between lattice spin operators and continuum fields is

$$
\hat{S}_{j}^{a} \sim \hat{M}^{a}(x)+(-1)^{j} \hat{n}^{a}(x)
$$

where $x=j a_{0}$ ( $a_{0}$ is the lattice spacing). The smooth components of the spin operators are proportional to the currents $\hat{M}^{a}(x) \propto \hat{J}^{a}(x)$, while $\hat{n}^{a}(x)$ are expressed in terms of Ising order and disorder operators as

$$
\begin{aligned}
& \hat{n}^{x}(x) \propto \hat{\sigma}^{1}(x) \hat{\mu}^{2}(x) \hat{\mu}^{3}(x), \\
& \hat{n}^{y}(x) \propto \hat{\mu}^{1}(x) \hat{\sigma}^{2}(x) \hat{\mu}^{3}(x), \\
& \hat{n}^{z}(x) \propto \hat{\mu}^{1}(x) \hat{\mu}^{2}(x) \hat{\sigma}^{3}(x) .
\end{aligned}
$$

In order to facilitate comparisons between field-theory and iDMRG results for the lattice model it is useful to define lattice operators

$$
\hat{m}_{j}^{\alpha}=\frac{\hat{S}_{j}^{\alpha}+\hat{S}_{j+1}^{\alpha}}{2}, \quad \hat{n}_{j}^{\alpha}=(-1)^{j} \frac{\hat{S}_{j}^{\alpha}-\hat{S}_{j+1}^{\alpha}}{2} .
$$

At long distances we have

$$
\hat{m}_{j}^{\alpha} \approx \hat{M}^{\alpha}(x), \quad \hat{n}_{j}^{\alpha} \approx \hat{n}^{\alpha}(x) .
$$

It is convenient to use the $\mathrm{U}(1)$ symmetry to bosonize

$$
\hat{L}_{1}+\mathrm{i} \hat{L}_{2} \sim \frac{1}{\sqrt{\pi a_{0}}} e^{-\mathrm{i} \sqrt{4 \pi} \hat{\varphi}_{L}}, \quad \hat{R}_{1}+\mathrm{i} \hat{R}_{2} \sim \frac{1}{\sqrt{\pi a_{0}}} e^{\mathrm{i} \sqrt{4 \pi} \hat{\varphi}_{R}} .
$$

In terms of the corresponding canonical Bose field $\hat{\Phi}=\hat{\varphi}_{L}+\hat{\varphi}_{R}$ and the dual field $\hat{\Theta}=\hat{\varphi}_{R}-\hat{\varphi}_{L}$ the field theory (5) reads:

$$
\begin{aligned}
\hat{\mathcal{H}} & =\hat{\mathcal{H}}_{3}+\hat{\mathcal{H}}_{B}+\hat{\mathcal{H}}_{\text {int }}, \\
\hat{\mathcal{H}}_{3} & =\frac{\mathrm{i} v_{3}}{2}\left[\hat{L}_{3} \partial_{x} \hat{L}_{3}-\hat{R}_{3} \partial_{x} \hat{R}_{3}\right]-\mathrm{i} m_{3} \hat{R}_{3} \hat{L}_{3}, \\
\hat{\mathcal{H}}_{B} & =\frac{v}{2}\left[\frac{1}{K}\left(\partial_{x} \hat{\Phi}\right)^{2}+K\left(\partial_{x} \hat{\Theta}\right)^{2}\right]-\frac{m}{\pi a_{0}} \cos \sqrt{4 \pi} \hat{\Phi}, \\
\hat{\mathcal{H}}_{\text {int }} & =\frac{2 \mathrm{i} g}{\pi a_{0}} \cos (\sqrt{4 \pi} \hat{\Phi}) \hat{L}_{3} \hat{R}_{3}+\lambda^{\prime} \sin (\sqrt{\pi} \hat{\Phi}) \hat{\sigma}^{3},
\end{aligned}
$$

where $K$ is the Luttinger liquid (LL) parameter. 


\subsection{Renormalization group analysis}

The most relevant perturbation is always the dimerization, and concomitantly at weak coupling the $\lambda^{\prime}$ term reaches strong coupling first under the renormalization group (RG) flow. This results in a non-zero dimerization

$$
\langle\hat{d}\rangle \equiv\left\langle\frac{1}{L} \sum_{j} \hat{D}_{j}\right\rangle \neq 0, \quad \hat{D}_{j}=(-1)^{j} \hat{\boldsymbol{S}}_{j} \cdot \hat{\boldsymbol{S}}_{j+1}
$$

For later convenience we define a lattice version of the normal-ordered dimerization operator

$$
\hat{d}_{j}=\frac{\hat{D}_{j}+\hat{D}_{j+1}}{2}-\langle\hat{d}\rangle .
$$

To see what happens after the dimerization perturbation has reached strong coupling we consider the next most relevant operators, which are the Majorana mass term and the costerm in the bosonic sector. Assuming that we have $m>0$, what happens then depends on the sign of the Majorana mass term $m_{3}$. If it is positive the third Ising model is in its disordered phase $\left\langle\hat{\sigma}^{3}(x)\right\rangle=0$, while $m_{3}<0$ implies that $\left\langle\hat{\sigma}^{3}(x)\right\rangle \neq 0$. In the latter case the strong coupling RG fixed point is amenable to a mean-field analysis. The term $\hat{\mathcal{H}}_{\text {int }}$ coupling the bosonic and fermionic sectors can be decoupled, e.g.

$$
\hat{\sigma}^{3}(x) \sin (\sqrt{\pi} \Phi(x)) \rightarrow\left\langle\hat{\sigma}^{3}(x)\right\rangle \sin (\sqrt{\pi} \hat{\Phi}(x))+\hat{\sigma}^{3}(x)\langle\sin (\sqrt{\pi} \hat{\Phi}(x))\rangle .
$$

This leads to a mean-field description in terms of an Ising model in a longitudinal field and a double sine-Gordon model 31,32

$$
\begin{aligned}
\hat{\mathcal{H}}_{\mathrm{MF}}= & \frac{\mathrm{i} v_{3}}{2}\left[\hat{L}_{3} \partial_{x} \hat{L}_{3}-\hat{R}_{3} \partial_{x} \hat{R}_{3}\right]-\mathrm{i} \widetilde{m}_{3} \hat{R}_{3} \hat{L}_{3}+h \hat{\sigma}^{3}+\frac{v}{2}\left[\frac{1}{K}\left(\partial_{x} \hat{\Phi}\right)^{2}+K\left(\partial_{x} \hat{\Theta}\right)^{2}\right] \\
& -\frac{\tilde{m}}{\pi a_{0}} \cos (\sqrt{4 \pi} \hat{\Phi})+\widetilde{\lambda} \sin (\sqrt{\pi} \hat{\Phi}),
\end{aligned}
$$

where

$$
\begin{aligned}
\tilde{\lambda} & =\lambda^{\prime}\left\langle\hat{\sigma}^{3}\right\rangle, \quad h=\lambda^{\prime}\langle\cos (\sqrt{4 \pi} \hat{\Phi})\rangle \\
\widetilde{m} & =m+2 i g\left\langle\hat{R}_{3} \hat{L}_{3}\right\rangle, \quad \widetilde{m_{3}}=m_{3}+\frac{2 g}{\pi a_{0}}\langle\cos (\sqrt{4 \pi} \hat{\Phi})\rangle .
\end{aligned}
$$

The classical ground state of the double sine-Gordon model is obtained by solving

$$
\frac{2 \widetilde{m}}{\pi} \sin \left(\sqrt{4 \pi} \hat{\Phi}_{c}\right)+\tilde{\lambda} \cos \left(\sqrt{\pi} \hat{\Phi}_{c}\right)=0
$$

Importantly, this tells us that for $\widetilde{m}>0$ we have

$$
\langle\cos (\sqrt{\pi} \hat{\Phi}(x))\rangle \neq 0
$$

which in turn implies that

$$
\left\langle\hat{n}^{z}(x)\right\rangle \propto\left\langle\hat{\sigma}^{3}(x) \cos (\sqrt{\pi} \hat{\Phi})\right\rangle \neq 0 .
$$

Hence the strong coupling RG fixed point describes a phase where antiferromagnetic order coexists with dimerization. This is the D-AFM phase identified above by the DMRG.

In the other phases the RG fixed points again occur at strong coupling but cannot be analyzed in terms of a simple mean-field argument. However, the field theory nevertheless allows for a description of the various transition lines as shown in what follows. 


\subsection{Quantum phase transitions}

\subsection{1 $\mathrm{D}-\mathrm{LD} \leftrightharpoons \mathrm{D}$-AFM phase transition line}

This corresponds to the situation where the bosonic sector remains gapped, while the third Ising model undergoes a transition between a disordered phase $\left\langle\hat{\sigma}^{3}\right\rangle=0$ on the D-LD side and an ordered phase $\left\langle\hat{\sigma}^{3}\right\rangle \neq 0$ on the D-AFM side of the phase diagram. As a result the D$\mathrm{LD} \leftrightharpoons \mathrm{D}-\mathrm{AFM}$ phase transition is in the universality class of the two-dimensional Ising model. In the vicinity of the transition we may project onto the low-energy Ising degrees of freedom following e.g. Ref. 33. Details are given in Appendix A. This yields

$$
\begin{aligned}
\left.\hat{m}_{j}^{z}\right|_{\text {low }} & =A \partial_{x} \hat{\sigma}^{3}(x)+\ldots, \\
\left.\hat{n}_{j}^{z}\right|_{\text {low }} & =B \hat{\sigma}^{3}(x)+\ldots, \\
\left.\hat{d}_{j}\right|_{\text {low }} & =\mathrm{i} C \hat{R}_{3}(x) \hat{L}_{3}(x)+\ldots
\end{aligned}
$$

Along the phase transition line we thus have

$$
\begin{aligned}
\left\langle\hat{n}_{j}^{z} \hat{n}_{j+\ell}^{z}\right\rangle & =B^{2} \ell^{-1 / 4}+\ldots, \\
\left\langle\hat{m}_{j}^{z} \hat{n}_{j+\ell}^{z}\right\rangle & =-\frac{A B}{4} \ell^{-5 / 4}+\ldots, \\
\left\langle\hat{m}_{j}^{z} \hat{m}_{j+\ell}^{z}\right\rangle & =\frac{5 A^{2}}{16} \ell^{-9 / 4}+\ldots,
\end{aligned}
$$

and

$$
\left\langle\hat{d}_{j} \hat{d}_{j+\ell}\right\rangle=C^{2} \ell^{-2}+\ldots
$$

The predictions $(29)-(32)$ are compared to iDMRG simulations below.

\subsection{2 $\mathrm{D}-\mathrm{H} \leftrightharpoons \mathrm{D}-\mathrm{AFM}$ phase transition line}

The D-AFM to D-H transition is described by the same scenario as discussed above, since it also belongs to the Ising universality class with $c=1 / 2$. Accordingly, Eqs. (29) $-(32)$ are valid on this transition line as well.

\subsection{3 $\mathrm{D}-\mathrm{H} \leftrightharpoons \mathrm{D}-\mathrm{LD}$ phase transition line}

As we cross from the D-AFM into the D-H phase at fixed $\Delta$ by increasing $D$ the (effective) Majorana mass $m_{3}$ increases. Assuming that this relation continues to hold, the characteristic energy scale in the Majorana sector can eventually become large compared to that of the bosonic sector and it is then justified to integrate out the Majorana sector. This leads to an effective low-energy description in terms of a sine-Gordon model

$$
\hat{\mathcal{H}}_{\text {low }}=\frac{v}{2}\left[\frac{1}{K}\left(\partial_{x} \hat{\Phi}\right)^{2}+K\left(\partial_{x} \hat{\Theta}\right)^{2}\right]-\frac{m^{*}}{\pi a_{0}} \cos (\sqrt{4 \pi} \hat{\Phi}) .
$$

The main effect of integrating out the Majorana sector is the renormalization of the sineGordon coupling. Importantly, $m^{*}$ can vanish for particular values of $D$, which corresponds 
to a phase transition line described by a LL characterized by the LL parameter $K$. The low-energy projections of the lattice spin operators along this line are

$$
\begin{aligned}
\left.\hat{d}_{j}\right|_{\text {low }} & =A_{D} \cos (\sqrt{4 \pi} \hat{\Phi}(x))+\ldots, \\
\left.\hat{n}_{j}^{z}\right|_{\text {low }} & =A_{z} \sin (\sqrt{4 \pi} \hat{\Phi}(x))+\ldots, \\
\left.\hat{n}_{j}^{x}\right|_{\text {low }} & =A_{x} \cos (\sqrt{\pi} \hat{\Theta}(x))+\ldots, \\
\left.\left(S_{j}^{+}\right)^{2}\right|_{\text {low }} & =A_{2} e^{\mathrm{i} \sqrt{4 \pi} \hat{\Theta}(x)}+\ldots \\
\left.\hat{m}_{j}^{x}\right|_{\text {low }} & =\frac{a_{0}}{\sqrt{\pi}} \partial_{x} \hat{\Phi}(x)+\ldots
\end{aligned}
$$

This gives the following field-theory predictions for power-law decays of two-point functions

$$
\begin{aligned}
\left\langle\hat{n}_{j}^{z} \hat{n}_{j+\ell}^{z}\right\rangle & =\frac{A_{z}^{2}}{2} \ell^{-2 K}+\ldots, \\
\left\langle\hat{n}_{j}^{\alpha} \hat{n}_{j+\ell}^{\alpha}\right\rangle & =\frac{A_{x}^{2}}{2} \ell^{-1 / 2 K}+\ldots, \quad \alpha=x, y, \\
\left\langle\left(\hat{S}_{j}^{+}\right)^{2}\left(\hat{S}_{j+\ell}^{-}\right)^{2}\right\rangle & =A_{2}^{2} \ell^{-2 / K}+\ldots, \\
\left\langle\hat{m}_{j}^{z} \hat{m}_{j+\ell}^{z}\right\rangle & =\frac{K}{2 \pi^{2}} \ell^{-2}+\ldots, \\
\left\langle\hat{d}_{j} \hat{d}_{j+\ell}\right\rangle & =\frac{A_{D}^{2}}{2} \ell^{-2 K}+\ldots
\end{aligned}
$$

\section{DMRG analysis}

In this section, we examine various two-point correlation functions of the lattice Hamiltonian (2) using iDMRG in order to prove the field-theory predictions described in the last section. Then, the topological properties of each phase are discussed by simulating topological order parameters.

\subsection{Phase transition lines}

\subsubsection{D-LD $\leftrightharpoons \mathrm{D}$-AFM and $\mathrm{D}-\mathrm{H} \leftrightharpoons \mathrm{D}$-AFM Ising phase transition lines}

For fixed $D / J=3$ and $\delta=0.1$ the Ising QPT occurs at $\Delta_{\mathrm{c}} \simeq 3.303$ between D-LD and DAFM phases as extracted from correlation length $\xi_{\chi}$. At this transition point various two-point functions can be computed by iDMRG. Here, $\chi=1600$. As shown in Fig. 2(a) field-theory predictions for diverse two-point functions of $z$-component spin operators (29)-(31) can be proved by iDMRG. Figure 2(b) demonstrates that also the dimer-dimer correlation function is in agreement with the power-law behavior predicted by Eq. (32) for large distances $\ell \gg 1$.

The relations between the coefficients in Eqs. 229 - 32 can be verified by fitting the iDMRG data to the field-theory predictions. For instance, in the case of the $\mathrm{D}-\mathrm{LD} \leftrightharpoons \mathrm{D}$ AFM transition at $D / J=3$ [Fig. 2(a)], we obtain $c_{1} \simeq 0.381(B \simeq 0.617)$ and $c_{3} \simeq 0.158$ ( $A \simeq 0.711$ ), i.e., $A B / 4 \simeq 0.110$, which is in good agreement with $c_{2} \simeq 0.114$ from Eq. 30. 

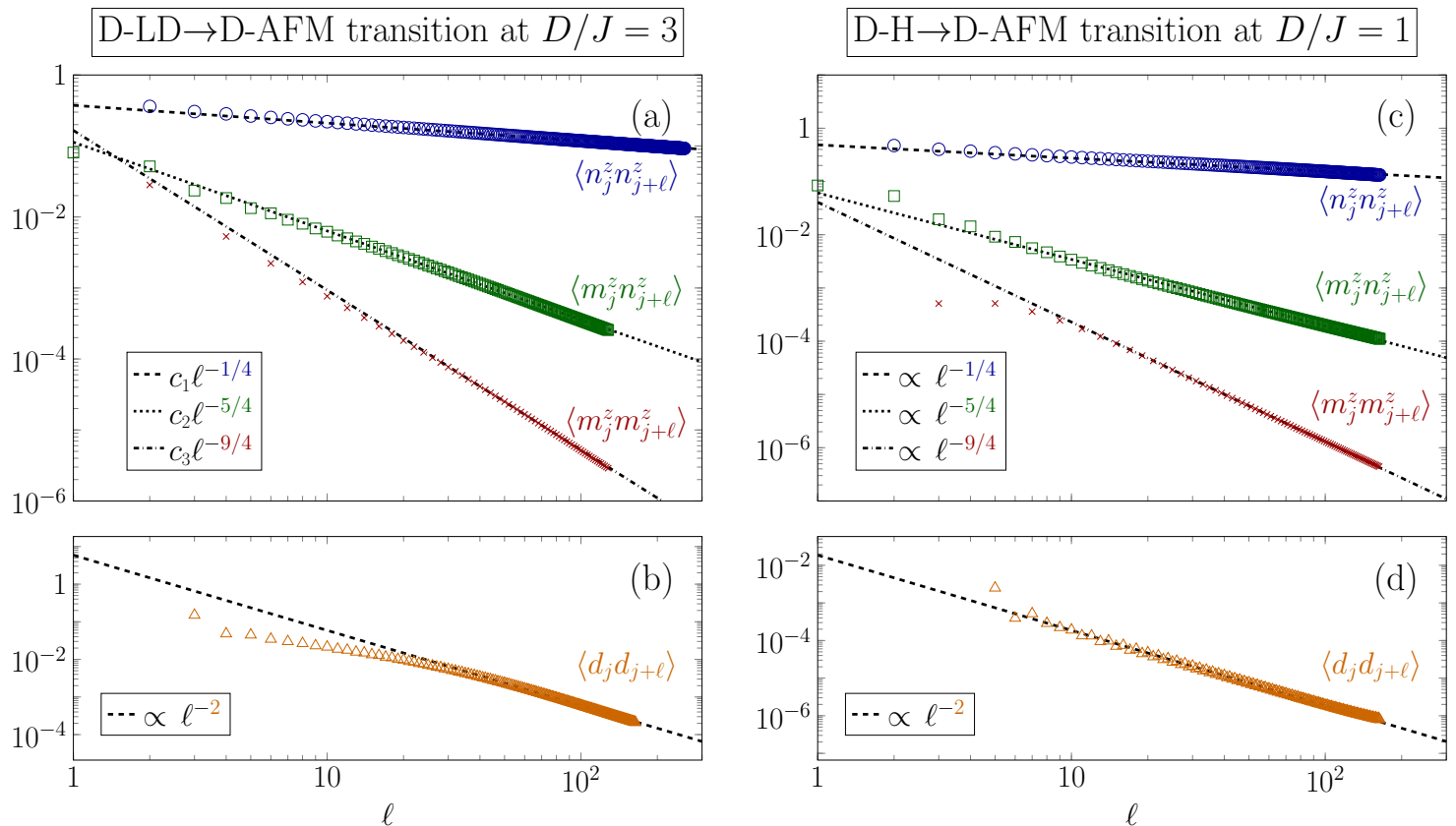

Figure 2: The connected longitudinal spin-spin (upper panels) and dimerization (lower panels) two-point functions at the Ising transition point for fixed $D / J=3$ (left panels) and $D / J=1$ (right panels) with $\delta=0.1$, obtained by iDMRG with $\chi=1600$. Correlation functions (symbols) show a power-law decay in accordance with the field-theory predictions Eqs. (29) (32) [lines].

Along the Ising critical line separating the D-H and D-AFM phases the long-distance behavior of these correlation functions determined by iDMRG is again in excellent agreement with field-theory predictions, $c f$. Eqs. (29)-(32). A representative example is shown in Figs. 2(c) and (d) for $D / J=1$ and $\Delta_{\mathrm{c}} \simeq 1.789$.

\subsection{2 $\mathrm{D}-\mathrm{H} \leftrightharpoons \mathrm{D}-\mathrm{LD}$ phase transition line}

Along the line of Gaussian QPTs separating the D-H and D-LD phases the exponents characterizing the long-distance behavior of correlation functions depends on the LL parameter $K$ as described in Eqs. (39)-(41) and (43). In order to facilitate a comparison to the field-theory results we therefore require the LL parameter $K$. For fixed $D / J=1$ the Gaussian transition occurs at $\Delta_{c} \simeq 1.135$. In Figs. 3 (a) and (b) we show numerical results of correlation functions obtained by iDMRG. The values of LL parameters extracted from the fits to Eqs. (39)-(41) and (43) show reasonable agreement with each other.

These values can also be extracted from the long-distance behavior of the smooth part of the spin-spin correlation function (42), that is, the LL parameter determines the amplitude of the correlation function but not the exponent. We calculate the longitudinal spin correlation function and isolate the smooth component from a Fourier transformed structure factor:

$$
S(q)=\frac{1}{L} \sum_{j \ell} e^{\mathrm{i} q(j-\ell)}\left(\left\langle\hat{S}_{j}^{z} \hat{S}_{\ell}^{z}\right\rangle-\left\langle\hat{S}_{j}^{z}\right\rangle\left\langle\hat{S}_{\ell}^{z}\right\rangle\right)
$$

for $q \approx 0$, where $q=2 \pi / L$. The LL parameter is determined as $K=\lim _{q \rightarrow 0} \pi S(q) / q[34$. 

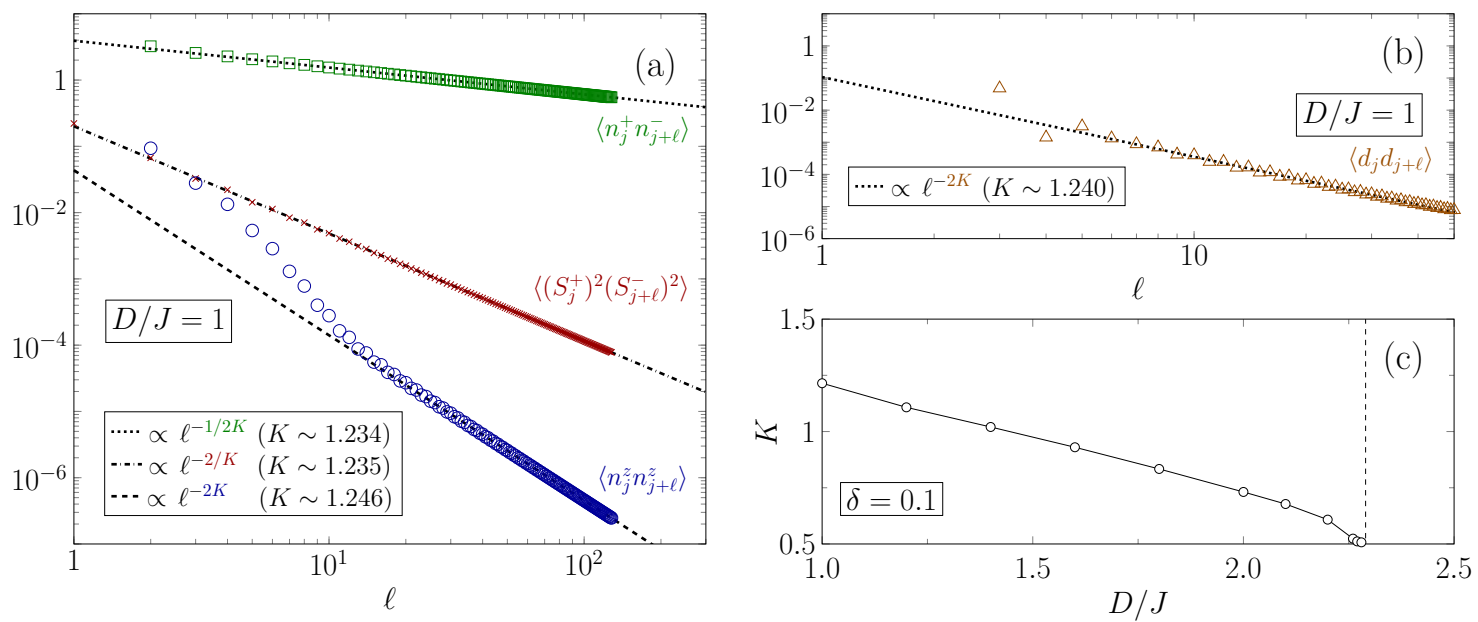

Figure 3: Spin-spin (a) and dimer-dimer (b) correlation functions at the $c=1$ transition for $D / J=1$ and $\delta=0.1$ computed by iDMRG with bond dimension $\chi=1600$. The extracted values of the LL parameter $K$ show good agreement with one other. (c) Extrapolated values of LL parameters $K$ via $S(q)$ of Eq. (44) on the $c=1$ transition line for $\delta=0.1$, obtained by DMRG with up to $L=512$ sites and open boundary conditions.

Figure 3(c) shows the results for the Luttinger parameter $K$ on the $c=1$ line for $\delta=0.1$. At $\Delta=1$ we have $K=1.215$, in reasonable agreement with the values obtained from the exponents of correlation functions in Figs. 3(a) and (b). Following the Gaussian transition line by increasing $\Delta$ and $D / J$ the Luttinger parameter decreases and takes the value $K \simeq 1 / 2$ at the point when the Gaussian line merges with the line of Ising QPTs.

\subsection{Topological order parameters}

Let us now the explore topological properties of each phase of the model (2). Following Vidal [35], we use the infinite matrix-product-state representation formed by $\chi \times \chi$ matrices $\Gamma_{\sigma}$ and a positive real, diagonal matrix $\Lambda$ :

$$
|\psi\rangle=\sum_{\cdots \sigma_{j}, \sigma_{j+1} \cdots} \cdots \Lambda \Gamma_{\sigma_{j}} \Lambda \Gamma_{\sigma_{j+1}} \cdots\left|\cdots \sigma_{j}, \sigma_{j+1}, \cdots\right\rangle
$$

where the index $\sigma$ labels the basis states of the local Hilbert spaces. The $\Gamma_{\sigma}$ and $\Lambda$ are assumed to be in the canonical form:

$$
\sum_{\sigma} \Gamma_{\sigma} \Lambda^{2} \Gamma_{\sigma}^{\dagger}=\mathbb{1}=\sum_{\sigma} \Gamma_{\sigma}^{\dagger} \Lambda^{2} \Gamma_{\sigma}
$$

If $|\psi\rangle$ is invariant under an internal symmetry represented by a unitary matrix $\Sigma_{\sigma \sigma^{\prime}}$, then the transformed $\Gamma_{\sigma}$ matrices satisfy [5, 36]

$$
\sum_{\sigma^{\prime}} \Sigma_{\sigma \sigma^{\prime}} \Gamma_{\sigma^{\prime}}=e^{\mathrm{i} \theta} U^{\dagger} \Gamma_{\sigma} U
$$

Here $U$ is a unitary matrix that commutes with $\Lambda$, and $e^{i \theta}$ is a phase factor. In the case of time reversal symmetry (inversion symmetry), $\Gamma_{\sigma}$ on the left-hand side is replaced by its 
complex conjugate $\Gamma_{\sigma}^{\dagger}$ (its transpose $\Gamma_{\sigma}^{T}$ ). Exploiting the properties of the matrices $U$ each SPT phase can be classified [5]: In the case of time reversal (inverse) symmetry the matrices satisfy $U_{\mathcal{T}} U_{\mathcal{T}}^{*}= \pm \mathbb{1}\left(U_{\mathcal{I}} U_{\mathcal{I}}^{*}= \pm \mathbb{1}\right)$, and the sign can be used to distinguish different SPT phases. In presence of a $\mathbb{Z}_{2} \times \mathbb{Z}_{2}$ symmetry the order parameter is given by

$$
O_{\mathbb{Z}_{2} \times \mathbb{Z}_{2}}=\frac{1}{\chi} \operatorname{Tr}\left(U_{x} U_{z} U_{x}^{\dagger} U_{z}^{\dagger}\right)
$$

where we use the symmetry operations $\hat{R}^{x}=\exp \left(\mathrm{i} \pi \sum_{j} \hat{S}_{j}^{x}\right)$ and $\hat{R}^{z}=\exp \left(\mathrm{i} \pi \sum_{j} \hat{S}_{j}^{z}\right)$ to calculate $U_{x}$ and $U_{z}$.

Figure 4 shows the iDMRG results for the order parameters in case of inverse and $\mathbb{Z}_{2} \times \mathbb{Z}_{2}$ symmetries. If $U_{x}$ and $U_{z}$ commute $\left(O_{\mathbb{Z}_{2} \times \mathbb{Z}_{2}}=1\right)$, the system is in a trivial phase, i.e., a site-factorizable LD state, whereas if they anticommute $\left(O_{\mathbb{Z}_{2} \times \mathbb{Z}_{2}}=-1\right)$, the system realizes a non-trivial Haldane state. If the symmetry is broken, we set $O_{\mathbb{Z}_{2} \times \mathbb{Z}_{2}}=0$. Obviously, the order parameter $O_{\mathbb{Z}_{2} \times \mathbb{Z}_{2}}$ changes its sign only if a phase transition occurs between D-LD and D-H phases. $O_{\mathcal{I}}$ behaves similarly to $O_{\mathbb{Z}_{2} \times \mathbb{Z}_{2}}$, i.e., $O_{\mathcal{I}}= \pm 1$ for the two symmetric phases, and $O_{\mathcal{I}}=0$ in the D-AFM phase.

To summarize this subsection, dimerization does not affect the topological properties of the system (2), so that the D-H (D-LD) phase remains a non-trivial (trivial) SPT phase as in the system without dimerization (1).

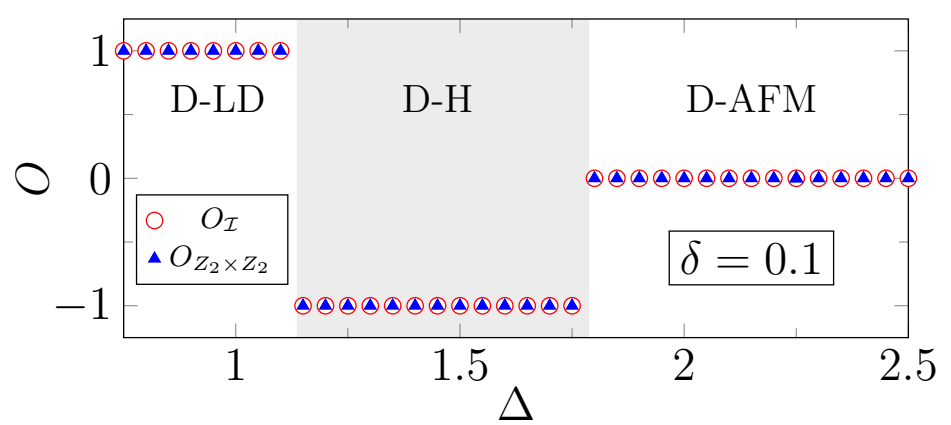

Figure 4: Topological order parameters for inversion symmetry $O_{\mathcal{I}}$ and $\mathbb{Z}_{2} \times \mathbb{Z}_{2}$ spin rotation symmetry $O_{\mathbb{Z}_{2} \times \mathbb{Z}_{2}}$ at $D / J=1$ and $\delta=0.1$.

\section{Relevance to experiments}

Let us finally relate our findings with experimental results. There are several realizations of spin-1 bond-alternating chains, such as $\mathrm{Ni}\left(\mathrm{C}_{9} \mathrm{H}_{2} 4 \mathrm{~N}_{4}\right)\left(\mathrm{NO}_{2}\right) \mathrm{ClO}_{4}$ [37, 38 and [Ni(333-tet) $(\mu$ $\left.\left.\mathrm{N}_{3}\right)_{n}\right]\left(\mathrm{ClO}_{4}\right)_{n}[39$ - 41 . Most remarkably, in the latter material a logarithmic decrease of the susceptibility was observed at low temperature, indicating a vanishing excitation gap [19]. Comparing quantum Monte-Carlo simulations with experimental data suggested that the material is described by a Hamiltonian of the form (2) with $\delta=0.25, \Delta=1$ and $D / J=0$. Totsuka et al. [42] determined the critical point for $D=0$ numerically and obtained $\delta_{\mathrm{c}}=$ $0.25 \pm 0.01$ and $c=1$, while results by Kitazawa and Nomura [11] suggested that $\delta_{\mathrm{c}}=0.2598$. Importantly these parameter sets are close to the location of the point where the Gaussian and Ising phase transitions merge [10,11]. 
In the following, we therefore determine the ground-state phase diagram of the model (2) for $\delta=0.25$ and reexamine the magnetic susceptibility of the above mentioned nickel compound using the infinite time-evolving block decimation (iTEBD) 35]. Figure 5(a) displays the corresponding phase diagram of the model (2). Although the extent of the Haldane phase is significantly reduced, the Gaussian and Ising transition lines can still be detected numerically. As shown in Fig. 5(b) the experimental data of the magnetic susceptibility for $\left[\mathrm{Ni}(333\right.$-tet $\left.)\left(\mu-\mathrm{N}_{3}\right)_{n}\right]\left(\mathrm{ClO}_{4}\right)_{n}$ can be fitted most successfully for $\Delta=1$ and $D / J=0.02$, taking the reported small single-ion anisotropy $D / J<0.1[19]$ into account. On the other hand, the numerical data at the Gaussian transition point for fixed $\Delta=1$ deviates from experimental ones in the lower-temperature regime. Thus, this nickel compound may be even closer to the Ising transition line than to the $c=1$ transition line than considered so far. It would be interesting to investigate signatures of the Ising QPT experimentally, e.g., by inelastic neutron scattering, where the corresponding dynamical structure factor can be calculated numerically, cf. Ref. [43.
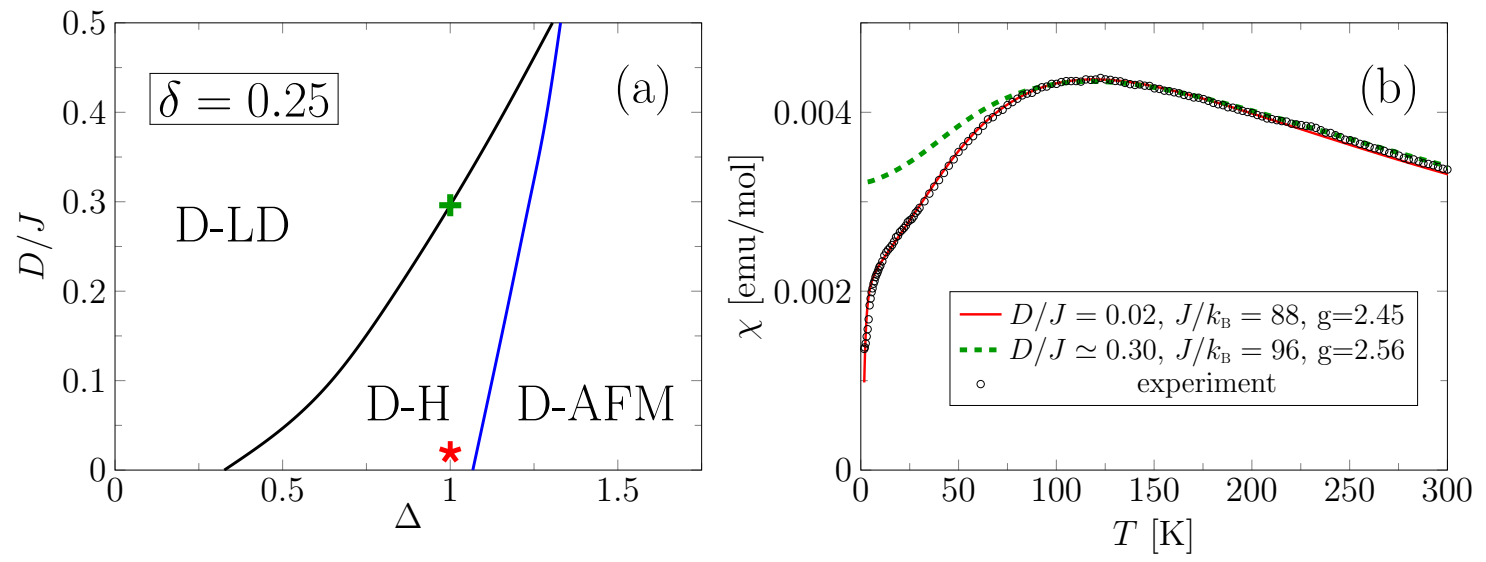

Figure 5: (a) Ground-state phase diagram of the model (2) for $\delta=0.25$. The red star denotes the parameter set corresponding to the $\mathrm{Ni}$ compound $\left[\mathrm{Ni}(333\right.$-tet $\left.)\left(\mu-\mathrm{N}_{3}\right)_{n}\right]\left(\mathrm{ClO}_{4}\right)_{n}$, and the green cross gives the Gaussian transition point $\left(D_{\mathrm{c}} \simeq 0.296\right)$ for fixed $\Delta=1$. (b) Temperature dependence of the magnetic susceptibility of the powdered sample for $[\mathrm{Ni}(333-$ tet) $\left.\left(\mu-\mathrm{N}_{3}\right)_{n}\right]\left(\mathrm{ClO}_{4}\right)_{n}$ (circles) taken from Ref. 19. The red solid line is the iTEBD data for $\Delta=1, D / J=0.02$ and $\delta=0.25$ with $J / k_{\mathrm{B}}=88$ and $g=2.45$. For comparison, we also show the iTEBD result at the Gaussian transition for fixed $\Delta=1$ (green dashed line).

\section{Summary and Conclusions}

In this work we investigated the ground-state phase diagram and quantum criticality of the dimerized spin-1 $X X Z$ chain with single-ion anisotropy $D$, employing a combination of analytical and numerical techniques. For weak dimerization $(\delta \lesssim 0.26)$ and single-ion anisotropy, the symmetry-protected topological Haldane phase survives and the transition between the D-LD and D-AFM phases, which is always of first order in the absence of dimerization, becomes partially continuous. The continuous section of the transition line belongs to the Ising universality class with central charge $c=1 / 2$. With increasing the magnitude of $D$, this Ising 
line terminates at a tricritical Ising point with $c=7 / 10$, above which the phase transition becomes first order. A comprehensive description of the phases and phase boundaries can be achieved by a bosonization-based field theory including three Majorana fermions. The field-theory predictions for various correlation functions have been confirmed by numerical iDMRG calculations.

Finally, we have revisited the experimental results for the $\mathrm{Ni}$ compound $[\mathrm{Ni}(333$-tet $)(\mu-$ $\left.\left.\mathrm{N}_{3}\right)_{n}\right]\left(\mathrm{ClO}_{4}\right)_{n}$ showing gapless behavior and have demonstrated that the corresponding parameter set might be not only in the vicinity of the Gaussian transition line but also very close to the Ising transition line. Further experimental research for this material, such as neutron scattering, would be desirable.

\section{Acknowledgements}

We thank M. Hagiwara for useful discussions and providing us with their experimental data. The iDMRG simulations were performed using the ITensor library. [44]

Funding information This work was supported by the Deutsche Forschungsgemeinschaft (Germany) under Grant No. FE 398/8-1 (FL), by the EPSRC under Grant No. EP/N01930X (FHLE) and the National Science Foundation under Grant No. NSF PHY-1748958 (FHLE). FHLE is grateful to the Erwin Schrödinger International Institute for Mathematics and Physics for hospitality and support during the programme on Quantum Paths. TY acknowledges support by a Chiba University SEEDS Fund and YO acknowledges support by a Grantin-Aid for Scientific Research (Grant No. 17K05530) from JSPS of Japan.

\section{A Low-energy projections of operators}

Let us denote the Euclidean action corresponding to the Hamiltonian density (14) by

$$
S=S_{3}+S_{B}+S_{\text {int }},
$$

where $S_{3}$ and $S_{B}$ involve only Ising and bosonic degrees of freedom respectively and $S_{\text {int }}$ describes the interaction between the two sectors. In the regimes where the mass scale associated with $S_{3}$ is much smaller (larger) than the one associated with $S_{B}$ and $S_{\text {int }}$ can be treated as a perturbation, we may integrate out the bosonic (fermionic) degrees of freedom, see e.g. Ref. [33].

\section{A.1 Integrating out the bosonic degrees of freedom}

This case pertains to the transition lines between the D-AFM phase and the D-LD and D-H phases. In these cases the low-energy projection of a general local operator is given by

$$
\left.\hat{O}\right|_{\text {low }}=\int \mathcal{D} \Phi e^{-S_{B}} e^{-S_{\text {int }}} \hat{O}=\langle\hat{O}\rangle_{\Phi}-\left\langle S_{\text {int }} \hat{O}\right\rangle_{\Phi}+\ldots
$$

where \langle\rangle$_{\Phi}$ denotes the average with respect to the bosonic action $S_{B}$. As we have assumed that the parameter $m$ is positive, we have

$$
\langle\sin (\sqrt{4 \pi} \Phi)\rangle_{\Phi}=0 .
$$


This implies that the low-energy projection of the dimerization operator is

$$
\begin{aligned}
\left.\hat{D}_{j}\right|_{\text {low }} & \sim-\left\langle S_{\text {int }} \hat{\sigma}^{3}(x) \sin (\sqrt{\pi} \hat{\Phi}(x))\right\rangle_{\Phi}+\ldots \\
& =-\lambda^{\prime} \int d \tau d y \hat{\sigma}^{3}(x) \hat{\sigma}^{3}(y, \tau)\langle\sin (\sqrt{\pi} \hat{\Phi}(x, 0)) \sin (\sqrt{\pi} \hat{\Phi}(y, \tau))\rangle_{\Phi}+\ldots \\
& =\langle\hat{d}\rangle+i C \hat{R}_{3}(x) \hat{L}_{3}(x)+\ldots
\end{aligned}
$$

In the last line we have used that the expectation value in the bosonic sector decays exponentially in the Euclidean distance $r=\sqrt{(x-y)^{2}+v^{2} \tau^{2}}$,

$$
\langle\sin (\sqrt{\pi} \hat{\Phi}(x, 0)) \sin (\sqrt{\pi} \hat{\Phi}(y, \tau))\rangle_{\Phi} \propto e^{-r / \xi}
$$

which in turn allows us to employ the operator product expansion in the Ising sector

$$
\hat{\sigma}^{3}(x) \hat{\sigma}^{3}(y, \tau)=\left(\frac{a_{0}}{r}\right)^{\frac{1}{4}}\left[1-\mathrm{i} \pi r \hat{R}_{3}(x) \hat{L}_{3}(x)\right]+\ldots
$$

Finally we have fixed the constant part in the low-energy projection by using that it must give the correct expectation value of the dimerization operator. Similarly we obtain

$$
\begin{aligned}
\left.\hat{M}_{j}^{z}\right|_{\text {low }} & \sim-\lambda^{\prime} \int d \tau d y \hat{\sigma}^{3}(y, \tau)\left\langle\partial_{x} \hat{\Phi}(x, 0) \sin (\sqrt{\pi} \hat{\Phi}(y, \tau))\right\rangle_{\Phi}+\ldots \\
& =A \partial_{x} \hat{\sigma}^{3}(x)+\ldots
\end{aligned}
$$

The leading contribution to the low-energy projection of $\hat{n}_{j}^{z}$ occurs at order $\hat{O}\left(\lambda^{\prime}\right)^{0}$ of our procedure and gives

$$
\begin{aligned}
\left.\hat{n}_{j}^{z}\right|_{\text {low }} & \sim B^{\prime}\langle\cos (\sqrt{\pi} \Phi \hat{(x)})\rangle_{\Phi} \hat{\sigma}^{3}(x)+\ldots \\
& =B \hat{\sigma}^{3}(x)+\ldots
\end{aligned}
$$

\section{A.2 Integrating out the fermionic degrees of freedom}

This case pertains to the transition line between the D-LD and D-H phases. Here we have

$$
\left.\hat{O}\right|_{\text {low }}=\int \mathcal{D} \hat{R}_{3} \mathcal{D} \hat{L}_{3} e^{-S_{3}-S_{\mathrm{int}}} \hat{O}=\langle\hat{O}\rangle_{3}-\left\langle S_{\mathrm{int}} \hat{O}\right\rangle_{3}+\ldots
$$

where \langle\rangle$_{3}$ denotes the average with respect to the Majorana action $S_{3}$. On the transition line we have $m_{3}>0$ which implies

$$
\left\langle\hat{\mu}^{3}(x)\right\rangle_{3} \neq 0 .
$$

An immediate consequence of $(58)$ is that

$$
\left.\hat{n}_{j}^{x}\right|_{\text {low }} \sim \cos (\sqrt{\pi} \hat{\Theta}(x))\left\langle\hat{\mu}^{3}(x)\right\rangle_{3}+\ldots
$$

The low-energy projections of other operators can be worked out as before

$$
\begin{aligned}
\left.\hat{n}_{j}^{z}\right|_{\text {low }} & \sim-\lambda^{\prime} B^{\prime} \int d y d \tau\left\langle\hat{\sigma}^{3}(x, 0) \hat{\sigma}^{3}(y, \tau)\right\rangle_{3} \sin (\sqrt{\pi} \hat{\Phi}(x, 0)) \cos (\sqrt{\pi} \hat{\Phi}(y, \tau)) \\
& =A_{z} \sin (\sqrt{4 \pi} \hat{\Phi}(x))+\ldots
\end{aligned}
$$



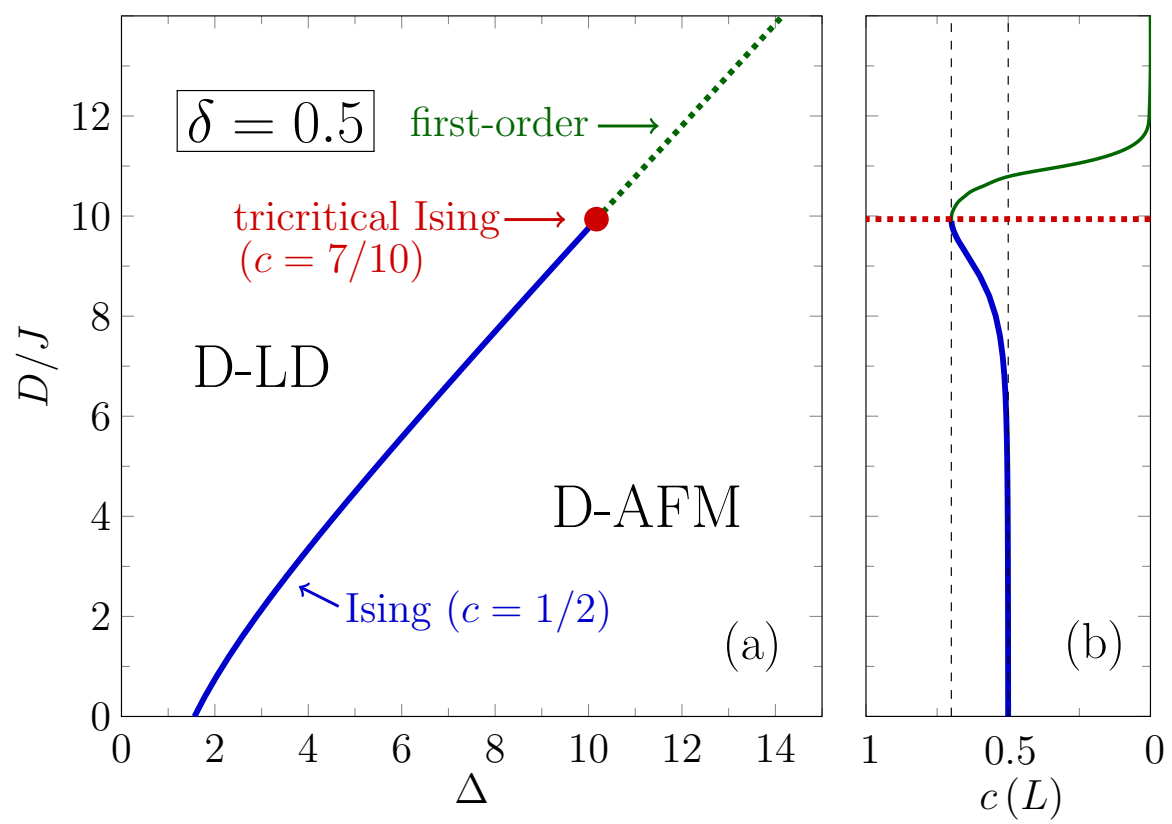

Figure 6: (a) Phase diagram of the model (2) for $\delta=0.5$. D-LD $\leftrightharpoons \mathrm{D}-\mathrm{AFM}$ phase boundary of the continuous Ising transition terminates at a tricritical Ising point. Beyond this point, the QPT becomes first order. (b) Central charge $c(L)$ on the D-LD $\leftrightharpoons \mathrm{D}$-AFM phase boundaries obtained numerically for $L=128$ and periodic boundary conditions.

Here we have used that

$$
\left\langle\hat{\sigma}^{3}(x, 0) \hat{\sigma}^{3}(y, \tau)\right\rangle_{3} \propto e^{-r / \zeta}
$$

which permits us to employ operator product expansions in the bosonic sector. The projection of the dimerization operator is

$$
\begin{aligned}
\left.\hat{D}_{j}\right|_{\text {low }} & \sim-\lambda^{\prime} \int d \tau d y\left\langle\hat{\sigma}^{3}(x) \hat{\sigma}^{3}(y, \tau)\right\rangle_{3} \sin (\sqrt{\pi} \hat{\Phi}(x, 0)) \sin (\sqrt{\pi} \hat{\Phi}(y, \tau))+\ldots \\
& =\langle\hat{d}\rangle+D \cos (\sqrt{4 \pi} \hat{\Phi})+\ldots
\end{aligned}
$$

\section{B Ground-state phase diagram for strong dimerization}

With increasing dimerization the $\mathrm{D}-\mathrm{H}$ phase is reduced, and it disappears for $\delta \gtrsim 0.26$ [1] if we limit ourselves to the parameter region $J>0$ and $\delta>0$. Figure 6(a) for $\delta=0.5$ demonstrates such a situation consisting of only D-LD and D-AFM phases, separated by continuous and first-order transition lines. At the meeting of these transition lines the numerically obtained central charge indicates $c=7 / 10$ [Fig. 6(b)], suggesting that this point belongs to the tricritical Ising universality class. 


\section{References}

[1] F. D. M. Haldane, Nonlinear field theory of large-spin Heisenberg antiferromagnets: Semiclassically quantized solitons of the one-dimensional easy-axis Néel state, Phys. Rev. Lett. 50, 1153 (1983), doi:10.1103/PhysRevLett.50.1153.

[2] I. Affleck, T. Kennedy, E. H. Lieb and H. Tasaki, Rigorous results on valencebond ground states in antiferromagnets, Phys. Rev. Lett. 59, 799 (1987), doi:10.1103/PhysRevLett.59.799.

[3] I. Affleck, Quantum spin chains and the Haldane gap, J. Phys.: Condens. Matter 1, 3047 (1989), doi:10.1088/0953-8984/1/19/001.

[4] Z.-C. Gu and X.-G. Wen, Tensor-entanglement-filtering renormalization approach and symmetry-protected topological order, Phys. Rev. B 80, 155131 (2009), doi:10.1103/PhysRevB.80.155131.

[5] F. Pollmann, A. M. Turner, E. Berg and M. Oshikawa, Entanglement spectrum of a topological phase in one dimension, Phys. Rev. B 81, 064439 (2010), doi:10.1103/PhysRevB.81.064439.

[6] W. J. L. Buyers, R. M. Morra, R. L. Armstrong, M. J. Hogan, P. Gerlach and K. Hirakawa, Experimental evidence for the Haldane gap in a spin-1 nearly isotropic, antiferromagnetic chain, Phys. Rev. Lett. 56, 371 (1986), doi:10.1103/PhysRevLett.56.371.

[7] J. P. Renard, M. Verdaguer, L. P. Regnault, W. A. C. Erkelens, J. Rossat-Mignod and W. G. Stirling, Presumption for a quantum energy gap in the quasi-one-dimensional $\mathrm{S}=1$ Heisenberg antiferromagnet $\mathrm{Ni}\left(\mathrm{C}_{2} \mathrm{H}_{8} \mathrm{~N}_{2}\right)_{2} \mathrm{NO}_{2}\left(\mathrm{ClO}_{4}\right)$, Europhys. Lett. 3, 945 (1987), doi:10.1209/0295-5075/3/8/013.

[8] T. Delica, K. Kopinga, H. Leschke and K. K. Mon, Thermal properties of chains of antiferromagnetically coupled spins with $s=1:$ Numerical evidence of the Haldane gap at nonzero temperatures, Europhys. Lett. 15, 55 (1991), doi:10.1209/0295-5075/15/1/010.

[9] W. Chen, K. Hida and B. C. Sanctuary, Ground-state phase diagram of $S=1$ XXZ chains with uniaxial single-ion-type anisotropy, Phys. Rev. B 67, 104401 (2003), doi:10.1103/PhysRevB.67.104401.

[10] A. Kitazawa, K. Nomura and K. Okamoto, Phase diagram of $S=1$ bond-alternating XXZ chains, Phys. Rev. Lett. 76, 4038 (1996), doi:10.1103/PhysRevLett.76.4038.

[11] A. Kitazawa and K. Nomura, Critical properties of $S=1$ bond-alternating $X X Z$ chains and hidden $Z_{2} \times Z_{2}$ symmetry, J. Phys. Soc. Jpn. 66, 3944 (1997), doi:10.1143/JPSJ.66.3944.

[12] F. Lange, S. Ejima and H. Fehske, Criticality at the Haldane-insulator chargedensity-wave quantum phase transition, Phys. Rev. B 92, 041120 (2015), doi:10.1103/PhysRevB.92.041120.

[13] S. Ejima, F. H. L. Essler, F. Lange and H. Fehske, Ising tricriticality in the extended Hubbard model with bond dimerization, Phys. Rev. B 93, 235118 (2016), doi:10.1103/PhysRevB.93.235118. 
[14] S. Ejima, F. Lange, F. H. Essler and H. Fehske, Critical behavior of the extended Hubbard model with bond dimerization, Physica B: Condensed Matter 536, 474 (2018), doi:10.1016/j.physb.2017.09.001.

[15] D. Friedan, Z. Qiu and S. Shenker, Conformal invariance, unitarity, and critical exponents in two dimensions, Phys. Rev. Lett. 52, 1575 (1984), doi:10.1103/PhysRevLett.52.1575.

[16] D. Friedan, Z. Qiu and S. Shenker, Superconformal invariance in two dimensions and the tricritical Ising model, Phys. Lett. B 151(1), 37 (1985), doi:http://dx.doi.org/10.1016/0370-2693(85)90819-6.

[17] S. R. White, Density matrix formulation for quantum renormalization groups, Phys. Rev. Lett. 69, 2863 (1992), doi:10.1103/PhysRevLett.69.2863.

[18] U. Schollwöck, The density-matrix renormalization group in the age of matrix product states, Ann. Phys. 326(1), 96 (2011), doi:10.1016/j.aop.2010.09.012.

[19] M. Hagiwara, Y. Narumi, K. Kindo, M. Kohno, H. Nakano, R. Sato and M. Takahashi, Experimental verification of the gapless point in the $S=1$ antiferromagnetic bond alternating chain, Phys. Rev. Lett. 80, 1312 (1998), doi:10.1103/PhysRevLett.80.1312.

[20] I. P. McCulloch, URL http://arxiv.org/abs/0804.2509, arXiv:0804.2509.

[21] P. Calabrese and J. Cardy, Entanglement entropy and quantum field theory, J. Stat. Mech. (2004)(06), P06002, doi:10.1088/1742-5468/2004/06/P06002.

[22] S. Nishimoto, Tomonaga-Luttinger-liquid criticality: Numerical entanglement entropy approach, Phys. Rev. B 84, 195108 (2011), doi:10.1103/PhysRevB.84.195108.

[23] L. Takhtajan, The picture of low-lying excitations in the isotropic Heisenberg chain of arbitrary spins, Phys. Lett. A 87, 479 (1982), doi:10.1016/0375-9601(82)90764-2.

[24] H. Babujian, Exact solution of the one-dimensional isotropic Heisenberg chain with arbitrary spins S, Phys. Lett. B 90, 479 (1982), doi:10.1016/0375-9601(82)90403-0.

[25] A. M. Tsvelik, Field-theory treatment of the Heisenberg spin-1 chain, Phys. Rev. B 42, 10499 (1990), doi:10.1103/PhysRevB.42.10499.

[26] H. Schulz, Phase diagrams and correlation exponents for quantum spin chains of arbitrary spin quantum number, Phys. Rev. B 34, 6372 (1986), doi:10.1103/PhysRevB.34.6372.

[27] I. Affleck, Critical behaviour of $S U(n)$ quantum chains and topological non-linear $\sigma$ models, Nucl. Phys. B 305, 582 (1988), doi:10.1016/0550-3213(88)90117-4.

[28] C. Itoi and M.-H. Kato, Extended massless phase and the Haldane phase in a spin-1 isotropic antiferromagnetic chain, Phys. Rev. B 55, 8295 (1997), doi:10.1103/PhysRevB.55.8295.

[29] R. Assaraf, P. Azaria, M. Caffarel and P. Lecheminant, Metal-insulator transition in the one-dimensional SU(N) hubbard model, Phys. Rev. B 60, 2299 (1999), doi:10.1103/PhysRevB.55.8295. 
[30] S. R. Manmana, A. M. Läuchli, F. H. L. Essler and F. Mila, Phase diagram and continuous pair-unbinding transition of the bilinear-biquadratic $S=1$ Heisenberg chain in a magnetic field, Phys. Rev. B 83, 184433 (2011), doi:10.1103/PhysRevB.66.024412.

[31] M. Fabrizio, A. Gogolin and A. Nersesyan, Critical properties of the doublefrequency sine-Gordon model with applications, Nucl. Phys. B 580(3), 647 (2000), doi:10.1016/S0550-3213(00)00247-9.

[32] G. Delfino and G. Mussardo, Non-integrable aspects of the multi-frequency sine-Gordon model, Nucl. Phys. B 516(3), 675 (1998), doi:10.1016/S0550-3213(98)00063-7.

[33] Y.-J. Wang, F. H. L. Essler, M. Fabrizio and A. A. Nersesyan, Quantum criticalities in a two-leg antiferromagnetic $s=1 / 2$ ladder induced by a staggered magnetic field, Phys. Rev. B 66, 024412 (2002), doi:10.1103/PhysRevB.66.024412.

[34] S. Ejima, F. Gebhard and S. Nishimoto, Tomonaga-Luttinger parameters for doped mott insulators, Europhys. Lett. 70(4), 492 (2005), doi:10.1209/epl/i2005-10020-8.

[35] G. Vidal, Classical simulation of infinite-size quantum lattice systems in one spatial dimension, Phys. Rev. Lett. 98, 070201 (2007), doi:10.1103/PhysRevLett.98.070201.

[36] D. Pérez-García, M. M. Wolf, M. Sanz, F. Verstraete and J. I. Cirac, String order and symmetries in quantum spin lattices, Phys. Rev. Lett. 100, 167202 (2008), doi:10.1103/PhysRevLett.100.167202.

[37] A. Escuer, R. Vicente and X. Solans, Syntheses of several kinds of one-dimensional nickel(ii)-nitrito complexes: a new $\mu$-nitrito nickel(ii) alternating chain, J. Chem. Soc., Dalton Trans. 1997, 531 (1997), doi:10.1039/A603070J.

[38] M. Hagiwara, H. Tsujii, C. R. Rotundu, B. Andraka, Y. Takano, N. Tateiwa, T. C. Kobayashi, T. Suzuki and S. Suga, Tomonaga-Luttinger liquid in a quasi-one-dimensional $S=1$ antiferromagnet observed by specific heat measurements, Phys. Rev. Lett. 96, 147203 (2006), doi:10.1103/PhysRevLett.96.147203.

[39] R. Vicente, A. Escuer, J. Ribas and X. Solans, The first nickel(ii) alternating chain with two different end-to-end azido bridges, Inorg. Chem. 31(9), 1726 (1992), doi:10.1021/ic00035a041.

[40] A. Escuer, R. Vicente, J. Ribas, M. S. El Fallah, X. Solans and M. Font-Bardia, trans$\left[\mathrm{Ni}(333-\text { tet })\left(\mu-\mathrm{N}_{3}\right)\right]_{n}\left(\mathrm{ClO}_{4}\right)_{n}$ and cis-[Ni(333-tet $)\left(\mu-\left(N_{3}\right)\right]_{n}\left(\mathrm{PF}_{6}\right)_{n}$ : Two novel kinds of structural nickel(ii) chains with a single azido bridge. magnetic behavior of an alternating $S=1$ chain with $\alpha=0.46$, Inorg. Chem. 33(9), 1842 (1994), doi 10.1021/ic00087a019.

[41] A. Escuer, R. Vicente, X. Solans and M. Font-Bardia, Crystal structure and magnetic properties of $\left[\mathrm{Ni}_{2}(\mathrm{dpt})_{2}(\mu-\mathrm{ox})\left(\mu-N_{3}\right)_{n}\right]\left(\mathrm{PF}_{6}\right)_{n}$ : A new strategy to obtain $S=1$ alternating chains, Inorg. Chem. 33(26), 6007 (1994), doi:10.1021/ic00104a007.

[42] K. Totsuka, Y. Nishiyama, N. Hatano and M. Suzuki, Isotropic spin-1 chains with bond alternation: analytic and numerical studies, J. Phys.: Condens. Matter 7(25), 4895 (1995), doi:10.1088/0953-8984/7/25/014. 
[43] S. Ejima and H. Fehske, Comparative density-matrix renormalization group study of symmetry-protected topological phases in spin-1 chain and Bose-Hubbard models, Phys. Rev. B 91, 045121 (2015), doi:10.1103/PhysRevB.91.045121.

[44] http://itensor.org/. 